\title{
Vers la fin du secret bancaire dans les centres financiers offshore : Une question d'éthique et de concurrence fiscale ${ }^{1}$
}

\author{
Arnaud Bourgain \\ CREA, Université du Luxembourg \\ 3 décembre 2014
}

\begin{abstract}
Résumé
Cette contribution présente différentes analyses du secret bancaire et de sa fin probable dans les centres financiers offshore selon deux points de vue complémentaires. D'une part, l'existence du secret bancaire est une question éthique qui soulève le problème du respect de la confidentialité, de l'égalité devant l'impôt et de l'évasion fiscale mais aussi du soutien à des systèmes économiques fondés sur l'opacité et la captation de rentes. Parallèlement, la transmission d'informations à des fins fiscales est analysée par quelques modèles économiques récents qui permettent d'envisager des effets indirects et relativement complexes d'un changement des conditions de la concurrence internationale pour l'attrait de capitaux. Ces deux approches complémentaires peuvent s'enrichir l'une de l'autre.
\end{abstract}

Mots clés : Centres offshore, secret bancaire, optimisation fiscale, évasion fiscale

\begin{abstract}
This paper is a survey of the literature that studies the end of bank secrecy in offshore financial centers. Research in this area can be read under two perspectives. On the one hand, the existence of the bank secrecy raises ethical issues like the respect of confidentiality, the equality of all before the taxation, tax evasion and the support for corrupted regimes. On the other hand, bank secrecy and its removal affect international tax competition among nation states and thus the incentives of exchange of information. A critical analysis of the existing contributions along these two strands enables us to understand better indirect and complex effects of the recent reforms on international competition for capital.
\end{abstract}

KEY WORDS: Offshore centers, bank secrecy, tax planning, tax evasion

JEL classifications: F21, H26, H87

\section{Introduction}

Depuis le milieu des années quatre-vingt-dix, plusieurs initiatives ont été lancées contre l'évasion fiscale internationale notamment permise par le secret bancaire dans les centres financiers offshore (OFCs). (OECD 1998, FSF 2000). Cette pression s'est intensifiée lors de la crise financière internationale (Saint-Amans, 2014) avec la signature de plus d'une centaine d'accords d'échange d'informations à des fins fiscales entre pays OCDE et non OCDE. Puis, en 2013, les Etats-Unis sont parvenus à faire signer à la Suisse un accord FATCA (Foreing Account Tax Compliance Act). En avril et mai 2013, des OFCs importants

\footnotetext{
${ }^{1}$ Je tiens remercier .... .
} 
(Luxembourg, Singapour, Iles Vierges Britanniques, Bermudes) ont annoncé leur acceptation d'introduire le principe d'échange automatique d'informations fiscales. Ce système de transmission devrait être effectif début 2017 pour une majorité de 89 pays volontaires. Pourtant, malgré une telle pression, les éléments empiriques ne permettent pas encore de conclure à une baisse de l'activité financière offshore. Les entités spéciales de type holding sont toujours plus nombreuses (au Luxembourg, le nombre de SOPARFI ${ }^{2}$ est passé de 2800 en 2000 à 55000 en 2011). De plus, Johannesen et Zucman (2014) ont constaté que les dépôts dans les OFCs ne se sont pas réduits significativement sur une période récente. Sur plusieurs points, la question du secret bancaire dans les centres offshore n'est pas simple.

Quel est le but recherché par l'abolition du secret bancaire, outre la lutte contre le blanchiment d'argent sale ? Est-ce davantage un principe d'éthique, d'égalité devant l'impôt, de coopération internationale face à l'évasion fiscale, ou un effort pour augmenter les recettes fiscales des pays à plus haute taxation (que nous appellerons ici " onshore ${ }^{3}$ ») ? Ces deux types d'objectifs ne sont pas antinomiques mais relèvent de domaines d'analyse différents.

L’objet de notre contribution est de présenter les analyses économiques récentes portant la fin du secret bancaire dans les centres offshore en distinguant successivement deux champs d'analyse : d'une part l'éthique et la morale, et d'autre part la concurrence fiscale internationale entre centres financiers.

Dans une première partie, nous montrons comment l'immoralité de l'évasion fiscale va, dans les années récentes, dominer les justifications traditionnelles de la confidentialité des investisseurs. En effet, les règles de confidentialité bancaire furent longtemps considérées comme des principes de protection de la vie privée des individus. C'est dans cette logique que le secret bancaire était légitimé dans les «havres » protecteurs vis-à-vis d'Etats prédateurs. Dans une optique radicalement opposée, l'ampleur de l'évasion fiscale internationale rompt le principe de l'égalité devant l'impôt, réduit les recettes fiscales des Etats, et finalement favorise une mauvaise gouvernance en permettant la fuite de capitaux issus de la corruption.

Une seconde partie est consacrée aux traitements de la question de l'évasion fiscale à travers les OFCs dans un cadre de concurrence pour l'attrait de capitaux. C'est dans ce contexte que Slemrod et Wilson (2009) démontrent l'effet parasitaire des OFCs qui fournissent des services à des fins d'évasion fiscale alors que d'autres auteurs (Hong et Smart 2010 ; Desai et al 2006) soulignent les effets bénéfiques de l'utilisation des instruments financiers offshore par les multinationales. Des contributions plus récentes (notamment Elsayyad et Konrad 2012 ; Bourgain, Pieretti et Zanaj 2014) envisagent et modélisent des effets concurrentiels plus complexes. Ils analysent comment la lutte contre les OFCs, particulièrement en imposant la transmission d'informations, modifie la concurrence entre centres offshore. Il est tenu compte du fait que les centres offshore sont capables d'offrir des

\footnotetext{
${ }^{2}$ Société de Participations Financières

${ }^{3}$ Le terme " onshore » peut être défini par opposition au terme offshore, c'est-à-dire des places ayant une clientèle domestique substantielle et qui n'offrent pas des conditions règlementaires et fiscales particulièrement avantageuses pour l'attrait de capitaux ou la domiciliation de sociétés.
} 
méthodes sophistiquées d'optimisation fiscale, en substitution à l'évasion fiscale traditionnelle par le secret bancaire. Ces travaux sur la concurrence fiscale internationale, en suivant une approche plus positive que normative, permettent de mieux analyser la lutte contre l'évasion fiscale internationale. Par contre, ces approches prennent encore trop peu en compte les notions de réputation, de transparence et d'équité comme variable clé de leur modélisation.

\section{La fin du secret bancaire : une question d'éthique}

Les arguments justifiant ou au contraire contestant le secret bancaire se trouvent pleinement dans le champ de l'éthique. Cette confidentialité protégeant les investisseurs des Etats totalitaires ou fiscalement prédateurs est remise en question en raison des effets pervers du secret et de la mauvaise réputation qu'il engendre.

Le principe de la protection de la confidentialité

Historiquement, le secret bancaire suisse est considéré comme la référence incontournable (Chavagneux, Palan 2006), car la loi de 1934 (art. 47) de la Confédération a introduit des mesures pénales sévères pour le faire respecter. Cet exemple sera suivi pas la plupart des autres centres financiers offshore. Vu le contexte historique, les banquiers suisses, souvent bien relayés par des articles de presse ${ }^{4}$, ont présenté ce secret comme l'élément protecteur des avoirs des juifs et des autres minorités persécutées par le régime nazi. Il ferait ainsi figure de rempart de défense des individus face aux régimes totalitaires de toute période. L'historien suisse S. Guex (1999) remet en cause cette présentation mythique en rappelant d'une part que les banques suisses attiraient des capitaux grâce à la confidentialité au moins depuis la Révolution française et d'autre part que le secret a été renforcé dans des conditions bien particulières qui ont peu à voir avec la protection des persécutés de la guerre. En effet, lors de la crise de 1929, l'Etat fédéral suisse a dû soutenir massivement son système bancaire en proie à de graves difficultés. Alors qu'une loi de 1933 organisait le renforcement de la surveillance des établissements financiers, les banquiers suisses ont obtenu en contrepartie que le secret bancaire soit consolidé. Or, à la même époque, en 1932, des scandales d'évasion fiscale, impliquant deux mille personnalités françaises, ont conduit la France à exercer une pression sur quelques banques suisses, effarouchant un grand nombre de déposants. La défense de l'attractivité de la place financière suisse semble bien avoir été l'élément déterminant du renforcement du secret bancaire. Cette attractivité des capitaux fut à nouveau défendue avec un certain succès par la diplomatie helvétique à la fin de la seconde guerre mondiale face aux pressions des Alliés qui souhaitaient inspecter eux-mêmes les avoirs détenus en Suisse (Guex 1999).

A côté de cette référence historique, la confidentialité bancaire peut toujours être présentée comme un principe général de respect de la vie privée des individus, principe qui

\footnotetext{
${ }^{4}$ The Economist 17 fév. 1996, p 78.
} 
ne serait pas propre aux centres offshore ${ }^{5}$, ni même aux banques (Frei 2004). Il serait possible d'invoquer les droits fondamentaux (art. 8 de la Convention Européenne des Droits de l’Homme qui protège le respect de la vie privée) pour sauvegarder le secret bancaire comme les autres secrets professionnels (Young 2013). Cependant, un protocole additionnel à la Convention (1952), précise que « les dispositions précédentes ne portent pas atteinte au droit que possèdent les Etats de mettre en vigueur les lois qu'ils jugent nécessaires pour règlementer l'usage des biens ou pour assurer le paiement des impôts... ». De plus, c'est précisément sur le terrain des droits fondamentaux que S. B. Cohen (2013) ${ }^{6}$ attaque le secret bancaire par une contribution au titre provocateur: "Does Swiss banks secrecy violate international human rights ? ». En effet, il considère qu'en réduisant les ressources fiscales des pays victimes de la fuite des capitaux, le secret bancaire compromet l'accès aux besoins primaires prévu dans le Pacte des Nations Unies relatif aux Droits Economiques, Sociaux et Culturels. Tous les signataires, dont la Suisse et le Luxembourg, seraient engagés par ce principe même en dehors de leur territoire.

Par ailleurs, il semble que les attentats du 11 septembre 2001 perpétrés sur le sol américain aient fait bouger les lignes des défenseurs les plus zélés de la confidentialité, en tant que composante de la vie privée (Young, 2013, p. 139). Par exemple, immédiatement après ces événements, un membre de l'Heritage Foundation, sentant venir une remise en cause du secret en raison de la lutte contre le crime, mettait en garde en titrant : "Moneylaundering bill should target criminals, not low taxes ${ }^{7}$.

\section{Moralité fiscale}

Traditionnellement, les défenseurs du secret bancaire ont soutenu que l'évasion fiscale internationale était la conséquence de l'existence d'Etats prédateurs ou caractérisés par une gouvernance de mauvaise qualité. Par exemple, Frei (2004) écrit : "To prevent and combat tax evasion, the best way from a Swiss perspective is to operate a system that imposes moderate taxes, ensures efficient and appropriate utilization of government resources, and uses an efficient method of tax collection ». Ce genre d'argument, bien que polémique, a le mérite de poser la question des déterminants du comportement des contribuables dans le pays d’origine et de solliciter la littérature économique montrant une certaine endogénéité de l'évasion fiscale. Ainsi, à côté des facteurs classiques comme le niveau d’impôt et le risque encouru par la fraude, la «morale fiscale » a de l'importance. Cette morale fiscale dépend d'une part de facteurs économiquement exogènes qui seraient d'ordres démographiques, historiques, religieux, culturels... D’autre part, un certain nombre de facteurs peuvent être considérés comme endogènes au système économique (Feld and Frey 2007) :

\footnotetext{
${ }^{5}$ Antoine (1999, p 13) precise : « confidentiality in financial affairs, including bank confidentiality, is part of the wider concept of personal privacy and this relationship is one that has been recognized judicially (...) This view of financial privacy and bank confidentiality is shared by other non-offshore European countries ».

${ }^{6}$ Professor of Law, Georgetown University.

${ }^{7}$ D. J. Mitchell, Money-laundering bill should target criminals, not low taxes, Executive summary 1492, Roe Institute for Economic Policy Studies at The Heritage Foundation, October 16, 2001.
} 
- $\quad$ la contrepartie à la contribution fiscale en termes de biens et de services publics, et donc l'efficacité de la gestion publique ;

- les procédures qui mènent à cet équilibre, ou autrement dit, la perception d’équité du système fiscal ;

- $\quad$ les relations entre contribuables et l'administration fiscale.

A la suite d'une adaptation d'un modèle d'évasion fiscale, Dell'Anno (2009) teste économétriquement l'effet de la «perception de l'efficacité de l'Etat » mesuré par un indicateur synthétisant le respect de la loi et des indicateurs macroéconomiques (inflation, taux de chômage) sur le taux d'évasion fiscale dans un panel de pays d’Amérique latine. Cette variable s'avère avoir un plus grand effet sur l'évasion fiscale que le niveau du taux d’imposition marginal ou qu'un indicateur de flexibilité du marché du travail. Le problème de morale peut donc être aussi recherché du côté de l'efficacité et de l'équité des politiques fiscales de certains Etats, et il ne suffit pas que les prélèvements soient légaux pour les rendre moraux (Bagus et al. 2011).

\section{Immoralité de l'évasion fiscale internationale}

Face à ces questionnements sur l'aspect moral de l'évasion fiscale, la forte pression contre le secret bancaire des centres offshore repose en grande partie sur des arguments moraux et politiques.

L'élément le plus grave en matière d'éthique est le lien, reconnu depuis de nombreuses années, entre le secret bancaire dans les OFCs et l'argent du crime et son blanchiment ${ }^{8}$. Nous n’insisterons pas sur cet aspect car les autorités des OFCs proclament leur soutien sans réserve à la lutte contre le blanchiment et à la levée du secret bancaire dans les affaires criminelles, même si le principe de double incrimination réduit ou allonge les délais de transmission d'informations dans le cadre de la coopération judiciaire internationale ${ }^{9}$. Par ailleurs, une distinction pourrait être soulignée entre l'évasion fiscale, illégale, utilisant largement le secret bancaire et l'optimisation fiscale employant des instruments légaux mais considérés dans une zone grise. Si d'un point de vue juridique cette distinction est primordiale, elle l'est beaucoup moins sur le plan moral. Ainsi, l'argumentation éthique contre le secret bancaire dans les OFCs et l'offensive internationale pour la transparence fiscale reposent essentiellement sur le problème politique d'égalité face à l'impôt et sur la dénonciation des effets de l'évasion fiscale internationale sur le sous-développement.

Le secret bancaire en facilitant l'évasion fiscale engendre des effets pervers sur la gouvernance publique qui peuvent se propager à l'ensemble de l'économie. La transparence de la politique fiscale est un pilier de la démocratie, car en étant visible aux yeux des

\footnotetext{
${ }^{8}$ La forte garantie de confidentialité ainsi que les instruments légaux de domiciliation dans les OFCs apparaissent dans la plupart des rapports internationaux (FMI, GAFI, FSF) comme un vecteur de blanchiment d’argent issus de la criminalité. Même avec des structures institutionnelles hautement réglementées, la nature même du secret bancaire rend très difficiles les enquêtes sur les transactions financières (Fitzgerald, 2004).

${ }^{9}$ Selon le principe de double incrimination pratiquée par certaines juridictions, les autorités judiciaires locales doivent prouver que l'acte faisant l'objet de l'enquête ait existé et qu'il constitue bien une infraction pénale selon la loi du pays requis s'il se produisait dans ce pays.
} 
citoyens, elle permet d'apporter une légitimité aux décisions fiscales gouvernementales. Comme le note Grinberg (2012, p. 357) en faisant référence notamment aux travaux de Mançur Olson ou de Douglass North : "A generation of economists, economic historians, sociologists, and political scientists has been influenced by the idea that relatively broadbased and transparent taxation, especially of mobile assets, generally tends to produce more representative government ». En effet, la transparence dans la taxation permet une négociation démocratique sur le partage de la charge fiscale entre les autorités publiques et les représentants des différents contribuables. Le secret bancaire va donc à l'encontre des intentions du législateur démocratiquement discutées (O’Neill, 2009). C’est dans cet esprit que l'OCDE, pour le titre de son célèbre rapport de 1998, a choisi le qualificatif d'« agressives » à propos des pratiques fiscales des OFCs.

La confidentialité bancaire permet aux bases fiscales les plus mobiles d'échapper à l'impôt et engendre deux effets politiques et économiques distorsifs. D'une part, elle implique une pression fiscale accrue sur les bases les moins mobiles comme le travail, et sur les petites entreprises. D’autre part, le secret assure la longévité des systèmes économiques fondés sur la captation de rentes par des groupes proches du pouvoir, en fournissant des instruments protecteurs de la corruption, en particulier dans les pays en développement ou émergents (notamment : Sikka 2003, van Dijk et al. 2006, Schjeldrup 2011).

C’est sans doute au sujet du développement que les arguments éthiques envers le secret bancaire ont eu une plus grande portée car l'évasion touche ici les pays les plus pauvres. Depuis les années 90, de grandes associations et réseaux internationaux ont mené l'offensive avec une abondance de rapports sur la fuite de capitaux des pays en développement facilitée par les OFCs : Global Financial Integrity, OXFAM ${ }^{10}$, Tax Justice Network, SOMO... Leur documentation sérieuse leur a permis d'être bien relayés dans les médias internationaux. En 2011, Tax Justice Network consacrait un rapport à l'Afrique, dans lequel les juridictions avec secret bancaire trouvaient bonne place. Généralement, des évaluations du stock de fuite de capitaux en provenance d'Afrique subsaharienne sont comparées avec sa dette extérieure ou l'aide publique au développement. Ces fuites pourraient représenter presque trois fois la dette extérieure sur la période 1970-2004. ${ }^{11}$ Le manque de recettes fiscales peut être aussi converti en hôpitaux, écoles... dans les rapports parlementaires ${ }^{12}$ pour bien souligner son caractère choquant.

Les effets sur la mauvaise gouvernance évoqués plus haut, sont bien sûr particulièrement évidents pour les pays en développement, et sont illustrés par une profusion de scandales de corruption où sont impliqués des OFCs. A côtés des banals cas de détournements, des montages plus sophistiqués sont permis par le secret des OFCs et vont assez loin dans l'immoralité. Ainsi, en matière d'endettement, N. Schaxson (2011) présente aussi la complicité des structures offshore opaques avec le détournement de prêts

\footnotetext{
${ }^{10}$ Oxfam. (2000). Tax Havens: Releasing the Hidden Billions for Poverty Eradication, London, Oxfam

${ }^{11}$ Ndikumana and Boyce, 2008, 4:1, Capital flight from Africa, TJN Focus.

${ }^{12}$ Sénat américain, Carl Levin, 18 juil. 2001.
} 
internationaux par une élite locale de pays en développement ou émergents. Plus précisément, certains fonds dits "vautours » sont constitués à partir de centres offshore pour acheter des emprunts publics à très forte prime de risque. Certains investisseurs sont intégrés dans ce type de fond pour leur position dans l'administration locale qui leur permettra d'user de leur influence pour que les dettes soient remboursées coûte que coûte. Le rôle des places offshore consiste à cacher cette participation à la population. Certes, les élites corrompues jouent en général le premier rôle, mais la présence du secret offshore leur est indispensable.

Dans le contexte actuel de remise en cause du secret bancaire, les plans sur l'échange d'informations risquent de laisser à l'écart les pays en développement. Dans les schémas en négociation, l'échange d'informations bancaires à des fins fiscales doit se faire sur une base réciproque. Or la plupart de pays en développement ne pourront pas consacrer des ressources nécessaires pour la collecte des informations requises selon les standards internationaux.

\section{Transmission d'informations à des fins fiscales et concurrence internationale pour l'attrait de capitaux}

La littérature économique récente sur le secret bancaire et les centres offshore n'est pas très abondante. Mais elle est intéressante car ces travaux posent en général le problème dans un cadre de concurrence internationale pour l'attrait de capitaux. Sans prétendre à l'exhaustivité, nous présentons quelques résultats de modélisation en nous concentrant sur trois questions essentielles: Comment démontrer le caractère parasitaire des centres offshore ? Comment modéliser les stratégies de transmission d'informations fiscales ? Et enfin, comment envisager la fin du secret bancaire dans un contexte de développement de l'optimisation fiscale?

Centres financiers offshore : des parasites?

Le modèle de Slemrod et Wilson (2009) est un modèle de concurrence fiscale standard incluant un paradis fiscal qui joue un rôle parasitaire sur les revenus créés dans les autres pays. Les auteurs partent d'hypothèses très négatives quant à l'activité des OFCs. Ainsi, il existe un marché mondial des services de dissimulation de revenus des capitaux, où l'offre est assurée par les OFCs. Ces services sont achetés par les firmes des pays à fiscalité normale, en plus des ressources réelles nécessaires à la production. De plus, les administrations fiscales des pays onshore doivent dépenser des ressources additionnelles dans la lutte contre l'évasion fiscale. Logiquement, la présence des OFCs intensifie la concurrence fiscale et induit une baisse des taux d'imposition des pays onshore et une réduction de l'offre de biens publics. Dans ce modèle, la présence des OFCs réduit le bien-être des individus essentiellement par deux canaux. Premièrement, les ressources utilisées pour la dissimulation fiscale ainsi que celles dépensées pour la lutte contre l'évasion sont des ressources prélevées sur la création de revenus productifs dans le pays onshore. Deuxièmement, les centres offshore entraînent des distorsions des politiques fiscales. En effet, les OFCs augmentent le coût marginal de fourniture des biens publics car l'accroissement de la fiscalité sur le capital, nécessaire pour financer les biens publics additionnels, va inciter les firmes à davantage 
utiliser les services des OFCs. Les gouvernements vont alors préférer la taxation sur le travail à la taxation sur le capital. Or le coût marginal du bien public ne devrait pas dépendre de la source de l'imposition, sur le capital ou sur le travail. Une taxation plus élevée du travail entraine une hausse du coût du bien public, commun aux deux sources d'imposition et donc une diminution de la fourniture de biens publics. L’élimination de ce marché pour la dissimulation apporterait alors une amélioration de bien-être.

Le modèle Slemrod et Wilson a le mérite de la netteté de ses résultats, mais qui sont sans surprise étant donné les hypothèses restrictives, uniquement négatives quant au rôle joué par les OFCs. Des travaux récents envisagent d'autres mécanismes ou d'autres hypothèses sur leurs rôles et obtiennent des résultats plus nuancés que ce soit en faisant intervenir les firmes multinationales (FMN) ou les placements financiers des investisseurs. Desai et al. (2006) ont montré empiriquement et théoriquement que des investissements dans les OFCs et dans les pays voisins non-OFCs pouvaient être complémentaires. Du côté de l'optimisation fiscale des FMN, Hong et Smart (2010) développent un modèle avec un centre offshore et une économie ouverte onshore. Celle-ci est composée de deux secteurs productifs : un secteur multinational (celui des FMN) qui utilise du travail et du capital mobile ; et un secteur domestique utilisant du travail et du capital entrepreneurial immobile. Comme dans d'autres modèles de concurrence fiscale, la présence de centre offshore fait baisser le taux effectif des FMN en leur permettant un transfert de revenu vers les OFCs pour un taux fiscal donné. La charge fiscale est alors davantage transférée sur les travailleurs locaux. Toutefois, le gouvernement peut organiser une redistribution fiscale à leur profit et au détriment des entrepreneurs locaux. Dans ce cas, une augmentation du taux de taxation des profits, dans les deux secteurs, ne fait pas fuir les FMN qui peuvent économiser une partie de leur charge fiscale grâce aux OFCs. Dans cette configuration, la présence d’OFCs pourrait améliorer le bien-être des individus dans le pays onshore. Par contre, le modèle ne tient pas compte des critiques morales envers une telle situation qui porte atteinte à l'équité fiscale. Les avantages accordés aux FMN rompent les principes d'égalité devant l'impôt et en général celui de la transparence des règles fiscales.

Cantonner les centres offshore dans un rôle de pure dissimulation peut paraître trop réducteur. C’est ainsi que Bucovetsky (2011), toujours à propos des firmes multinationales, évacue la recherche d'opacité ou de secret dans les OFCs pour lui substituer une garantie d'un régime fiscal favorable pour les activités facilement délocalisables des FMN (finance, services de navigation, services d'ingénierie internationale...). C’est alors la réputation des centres offshore pour la protection des investissements qui joue un rôle crucial et les résultats s'écartent sensiblement du modèle de Slemrod-Wilson. Dans une démarche parallèle, appliquée aux investissements financiers dans les OFCs en concurrence avec les pays nonOFCs, Pieretti, Thisse et Zanaj (2014), considèrent que la simple attractivité fiscale avec secret bancaire ne représente qu'une partie de la réalité des caractéristiques des OFCs. Les petits pays disposent en général d’un cadre institutionnel et légal plus flexible qui peut s'adapter facilement à un nouvel environnement pour attirer et protéger les investissements financiers. C'est sur cet avantage comparatif, dont le caractère exogène peut être critiqué, que repose l'essentiel du modèle. Ainsi, est envisagée une attractivité par deux instruments : le 
taux fiscal et les infrastructures institutionnelles. En exploitant l'avantage du premier instrument, l'OFCs se comporte en «tax-haven » et avec le second il est un « safe-haven »; les deux spécialités pouvant bien sûr coexister. Les résultats sont nuancés selon que les gouvernements sont de type prédateur (Leviathan) ou bienveillant. Avec des Etats bienveillants, d'un point du vue du bien-être, la concurrence en termes institutionnels est préférable à une simple concurrence fiscale ; et ceci avec ou sans le secret bancaire. Avec le secret bancaire, la concurrence fiscale entraine une désescalade fiscale et les infrastructures institutionnelles doivent être financées par d'autres sources que l'imposition des revenus du capital. Sans le secret bancaire, les Etats peuvent taxer les revenus du capital et se concurrencer en termes d'infrastructures institutionnelles. En fait, ajouter ce type de concurrence institutionnelle apporte des effets positifs qui compensent les effets dommageables d'une concurrence fiscale avec secret bancaire. Ces travaux s'appuient sur une connaissance plus fine et non caricaturale des activités des OFCs. Cependant, la substance des activités des OFCs non liées au secret et à l'évasion fiscale demande encore à être mesurée objectivement. Cette distinction n’est pas aisée et c'est précisément le sens d’un des récents rapports de l’OCDE (2014) visant les domiciliations sans substance.

\section{Stratégies pour instaurer la transmission d'informations}

Les « accords de Feira », nom de la ville portugaise où eu lieu le sommet européen de juin 2000, ont prévu que les pays européens devaient instaurer l'échange automatique d'informations sur les revenus (intérêts) de l'épargne, avec un période transitoire où les Etas pouvaient choisir un prélèvement à la source des non-résidents. C’est justement à cette période que quelques travaux ont modélisé le choix d'accepter volontairement des accords de transmission d'information. Bacchetta et Espinoza (2000) ont élaboré un modèle de jeux répétés pour comprendre dans quelles conditions deux pays, éventuellement de taille asymétrique, seraient incités à procéder à un échange d’information. Dans ce type de modèle, une clause de coopération sera adoptée si le traité est considéré comme durable après des interactions répétées entre les autorités fiscales. Les pays pourraient s’engager dans un comportement coopératif dans le cas où ils savent que s'écarter de l'échange d’informations mènerait les autres pays à appliquer la même politique dommageable. Les auteurs parviennent à définir des conditions dans lesquelles les pays n’ont pas intérêt à échanger l'information. C'est le cas notamment lorsque les flux de capitaux entrants sont plus sensibles à l'échange d'informations que les capitaux sortants, ou lorsque la fiscalité sur les résidents est basse comparée à la taxe sur les non résidents... Huizinga et Nielson (2003), avec une technique de modélisation proche, aboutissent à la solution qu'un grand pays va choisir l'échange d'informations alors qu'un petit pays opte pour le prélèvement à la source. Ce résultat est intéressant car il reproduit la décision, lors des Accords de Feira, du Luxembourg, de la Belgique et de l'Autriche de choisir le prélèvement alors que les autres pays européens, plus grands choisissaient l'échange d'information. Remarquons cependant que dans ces modèles de jeux répétés, les Etats, même avec des tailles asymétriques, sont censés suivre la même stratégie à chaque période. Cette hypothèse est particulièrement restrictive sachant qu'en réalité, les petits pays sont en général beaucoup plus flexibles et ont des groupes de pression proches du gouvernement. De plus, ces modèles ne font intervenir ni 
la réputation, ni aucune pression externe, éléments pourtant fondamentaux pour comprendre le comportement des OFCs.

Récemment, Elsayyad et Konrad (2012) ont proposé une modélisation qui correspond davantage à la dernière phase de lutte contre les activités offshore. En effet, à la suite de la crise financière de 2008, le G20 a décidé d'intensifier la pression pour la signature d'accords bilatéraux d'échange d'informations à des fins fiscales. L'apport essentiel du modèle d'Elsayyad et Konrad (2012) est d'envisager comment la lutte pour contraindre les OFCs à l'échange d'information, va modifier la concurrence entre les centres offshore pour l'attrait de capital mobile. Si les accords sont adoptés de façon séquentielle, les uns après les autres, la sortie de certains OFCs du marché des placements offshore va accroître la concentration de ce marché. Par conséquent, conformément aux analyses usuelles d’économie industrielle, les OFCs ayant réussi à se maintenir sur le marché vont devenir plus profitables, et donc plus résistantes à la pression internationale. Ainsi, une lutte séquentielle est sans doute plus facile à mener dans un premier temps, mais avec l'augmentation de la concentration, la situation peut être aggravée d'un point de vue des pays onshore, car non seulement la résistance sera plus forte, mais le montant des commissions transférées vers les OFCs sera accru. Les auteurs en arrivent à la conclusion qu'une action de type «big bang » serait préférable. Malgré le manque de travaux empiriques portant sur la situation concurrentielle des centres offshore, le mérite de ce travail est bien de traiter théoriquement cet aspect de la lutte contre le secret bancaire. Toutefois, le caractère assez binaire (existence ou disparition du centre offshore) de la problématique du modèle porte le flanc à la critique. En effet, il est généralement observé et il est assez logique que face à la pression internationale, les centres offshore ne disparaissent pas en tant que tels, mais se transforment, s'adaptent au nouvel environnement règlementaire, et donc restent dans le jeu concurrentiel. Cette évolution est justement à l'origine des travaux qui envisagent une mutation sous la forme de l'optimisation fiscale sans recours au secret bancaire.

\section{La fin du secret bancaire dans un contexte de développement de l'optimisation fiscale}

Il existe une abondance de preuves de l'essor des stratégies d'optimisation, sans secret bancaire, aujourd'hui accessibles aux investisseurs individuels aisés alors que ces techniques étaient souvent conçues pour les multinationales. Depuis une dizaine d'années, la presse spécialisée regorge de déclarations de fiscalistes comme celle du juriste new-yorkais K. Rubinstein (2010) proclamant : « secrecy has no place in proper tax planning... ». En effet, l'optimisation fiscale ${ }^{13}$ se développe essentiellement par l'utilisation d'entités spéciales offshores comme les différents holdings, trusts, sociétés de participations financières ... Ces entités appelées aussi "véhicules » permettent de minimiser la charge fiscale des investisseurs passant par les OFCs en exploitant efficacement, sans besoin du secret bancaire, les traités de non double imposition et la directive européenne « maison mère-filiale »

\footnotetext{
${ }^{13}$ L’OCDE (2009, p.21) résume parfaitement la situation : "Wealthy investors are often highly mobile and may be attracted to countries perceived as offering a favorable taxation environment. This may include such factors as no or low capital gains tax on the disposition of privately held assets and the presence of a good treaty-network. Rather than changing their tax residence, wealthy investors may hold investments through no or nominally taxed offshore entities with a view to mitigate tax on foreign source income or gains. »
} 
(1990). La lutte contre cette utilisation, pouvant être jugée abusive, n’est pas aisée car sa simple remise en cause pourrait porter atteinte à la libre circulation des capitaux, favorable aux investissements directs et prévue notamment dans les règles du Marché unique.

Observant le développement de l’optimisation fiscale par des techniques ne nécessitant pas le secret bancaire, Bourgain, Pieretti et Zanaj (2014) proposent une analyse de la concurrence entre OFCs et pays onshore dans l'attrait de capitaux qui envisage l'éventuelle substitution de l'optimisation fiscale à l'évasion fiscale. Ils montrent comment la fin du secret bancaire va modifier la situation concurrentielle entre les centres offshore et les pays onshore et finalement engendrer un développement de l'optimisation. Deux scénarios sont considérés. Dans le premier, le secret bancaire des OFCs permet l'évasion fiscale. Cependant, cette stratégie subit une pression internationale, sous forme par exemple de « listes noires », qui atteint la réputation de l'investisseur. Est-ce une prise en compte de l'éthique ou un moyen de défendre la base fiscale onshore ? En tout cas, cette pression est une variable essentielle du modèle, et elle est considérée comme endogène car dépendant positivement de l'intégration financière internationale. Dans un second scénario, l'OFC accepte d'échanger les informations à des fins fiscales; mais dans ce cas, des instruments sophistiqués d'optimisation fiscale sont disponibles pour diminuer la charge fiscale des investisseurs à travers l'OFC. La fin du secret bancaire élimine la pression internationale, mais il existe un risque propre à l'optimisation fiscale car celle-ci est fortement dépendante de règles légales qui peuvent être modifiées notamment à des fins anti-offshore. Un premier résultat obtenu par ce modèle est que la levée du secret bancaire peut accroître les recettes fiscales du pays onshore, mais seulement si les économies fiscales permises par l'optimisation n'excèdent pas une certaine proportion. Ce seuil dépend positivement du risque spécifique de l'optimisation fiscale, mais aussi du degré d'intégration financière internationale. L'intuition de ce dernier effet est un peu plus indirecte. Dans le modèle, l'accroissement du degré d'intégration a ainsi deux effets contraires sur les recettes fiscales du pays onshore dans le cas de secret bancaire. D'une part, il tend à diminuer les recettes fiscales car les contribuables deviennent moins captifs; d'autre part, les Etats onshore intensifient leur pression, ce qui peut augmenter les recettes fiscales. Cette seconde force peut devenir dominante lorsque la mobilité du capital est haute.

Par conséquent, ces résultats montrent qu’il est nécessaire de prendre davantage en compte les techniques d'optimisation fiscale qui se substituent de plus en plus au secret bancaire. En effet, les OFCs ne sont pas en activité ou simplement fermés, ils s'adaptent. Cette démarche est recommandée non seulement pour accroître les recettes fiscales des pays onshore, mais aussi pour inciter les OFCs à coopérer en échangeant les informations à des fins fiscales.

\section{Conclusion}

Traiter du secret bancaire dans les centres offshore selon deux approches, celle plus normative de l'éthique, et celle plus positive, de la concurrence pour l'attrait du capital mobile, apparaît indispensable pour bien appréhender les forces en jeu. En effet, ces 
dernières années, les problèmes moraux liés à l'évasion fiscale semblent avoir été déterminants dans l'abandon progressif du secret bancaire. Ces problèmes sont d'ailleurs aggravés lorsque la fuite de capitaux détériore la gouvernance dans les pays en développement, sachant que par causalité inverse la mauvaise gouvernance est aussi responsable de l'évasion. L'approche positive par la modélisation renforce considérablement la mise en évidence d'effets plus indirects ou plus complexes sur l'aspect plus ou moins parasitaire des OFCs, ou encore sur les conditions d'instauration de l'échange d'informations et surtout sur l'éventuelle substitution de l'optimisation fiscale à l'évasion fiscale. Il nous semble que ces deux approches pourraient être davantage intégrées. D’un point de vue théorique, la morale des contribuables a bien été intégrée dans les modèles économiques traitant du blanchiment d'argent (Masciandaro 2005) et de l'évasion fiscale en général. Mais le poids de l'éthique dans la concurrence internationale pour l'attrait de capitaux pourrait être davantage analysée et modélisée. D’un point de vue empirique, des travaux récents ont commencé à étudier les déterminants de l'échange d'informations fiscales entre pays (Elsayyad 2012, Bilicka and Fuest 2013). D’autres travaux économétriques devraient suivre

sur la prise en compte d'éléments éthique et de qualité de la gouvernance dans l'attrait de capitaux après la fin du secret bancaire.

\section{Références bibliographiques :}

Antoine R.-M.B., 1999, The protection of offshore confidentiality: policy implications and legal trends. Journal of Financial Crime, 7, 9-25

Bacchetta Ph., Espinoza M.P. (2000), Exchange of Information Clauses in International Tax Treaties, International Tax and Public Finance, 7, 275-293.

Bagus P., Block W., Eabrasu M., Howden D., Rostan J. (2011), The ethics of tax evasion, Business and Society Review, 116:3, 375-401.

Bilicka K., Fuest C. (2014), With which countries do tax havens share information? International Tax and Public Finance, 21, 175-197.

Bourgain A., Pieretti P. (2007), Discipliner les centres financiers offshore : Incitation par la pression internationale, Région et développement, 2007, n²6, 65-83.

Bourgain A., Pieretti P., Zanaj S. (2014), From evasion to tax planning, Discussion Paper, CREA.

Bucovetsky S. (2014), Honor among tax havens, Journal of Public Economics, 110, 74-81.

Chavagneux Ch., Palan R. (2006), Les paradis fiscaux, La Découverte, Paris.

Cohen S. B. (2013), Does Swiss Bank Secrecy Violate International Human Rights? Georgetown University Law Center.

Dell'Anno R. (2009), Tax evasion, tax morale and policy maker's effectiveness, The Journal of Socio-Economics, 38, 988-997. 
Desai M.A., Foley C.F., Hines J.R. (2006), The demand for tax haven operations. Journal of Public Economics, 90, 513-531.

Elsayyad M. (2012), Bargaining over Tax Information Exchange, Max Planck Institute for Tax Law and Public Finance, Working Paper 2012 - 02, February.

Elsayyad M., Konrad K.A. (2012), Fighting multiple tax havens, Journal of International Economics, Vol. 86 (2), 295-305.

Feld L.P., Frey B.S. (2007), Tax compliance as the result of a psychological tax contract: the role of incentives and responsive regulation, Law and Policy 29 (1), 102-120.

Financial Stability Forum, FSF. (2000), Report of the Working Group on Offshore Financial Centres, Report, 5 April.

FitzGerald V., (2004), Global Financial Information, Compliance Incentives and Terrorist Funding, European Journal of Political Economy, 20, 387-410.

Frei W. (2004), The protection of privacy and the protection of financial crime, Journal of Financial Crime, Vol 11, n² 4 , 397-398.

Grinberg I. (2012), The battle over taxing offshore accounts, UCLA Law Review, $\mathrm{n}^{\circ}$ 204, 304-383.

Guex S. (1999), Les origines du secret bancaire suisse et son rôle dans la politique de la Confédération au sortir de la Seconde Guerre mondiale, Genèses, 34, 4-27.

Hong Q., Smart M. (2010), In praise of Tax Havens: International Tax Planning and Foreign Direct. Investment, European Economic Review, 54(1), 82-95.

Huizinga H., Nielsen S.B. (2003), Withholding Taxes or Information Exchange: The Taxation of International Interest Flows, Journal of Public Economics 87 (1), 39-72.

Johannesen N., Zucman G. (2014), The End of Bank Secrecy? An Evaluation of the G20 Tax Haven Crackdown, American Economic Journal, February, 6 (1), 65-91.

Masciandaro, D. (2005), False and reluctant friends? National money laundering regulation. International compliance and non-cooperative countries. European Journal of Law and Economics 20, 17-30.

OCDE (2014), Lutter plus efficacement contre les pratiques fiscales dommageables, en prenant en compte la transparence et la substance, Projet OCDE/G20 sur l'érosion de la base d’imposition et le transfert de bénéfices, Éditions OCDE.

OECD (1998), Harmful Tax Competition: An Emerging Global Issue.

OECD (2009), Engaging with high net worth individuals on tax compliance, May.

O’Neill M. (2009), The social injustice of corporate tax avoidance, Tax Justice Focus, vol.5, $\mathrm{n}^{\circ} 2$, Tax Justice Network.

Palan (2002), Tax Havens and the Commercialization of State Sovereignty, International Organization, 51, 1, 151-176. 
Picard P., Pieretti P. (2011), Bank secrecy, illicit money and offshore financial centers, Journal of Public Economics, 95, 942-955.

Pieretti P., Thisse J.F., Zanaj S. (2014), Offshore financial centers and bank secrecy, CREA Discussion Paper, 2014-02, Luxembourg.

Saint-Amans P. (2014), Les progrès récents en matière de coopération dans le domaine de la lutte contre la fraude fiscale internationale, Rapport moral sur l'argent dans le monde, éd. Association d’Economie Financière.

Schjeldrup G. (dir),(2011), Tax havens and development, Report from the (Norway) Government Commission on Capital Flight from Poor Countries.

Shaxson N. (2012), Les paradis fiscaux, A. Versaille éditeur.

Sikka P. (2003), The role of offshore financial centres in globalization, Accounting Forum, 27:4, 365-399.

Slemrod J., Wilson J. (2009), Tax competition with parasitic tax havens, Journal of Public Economics 93 (11-12), 1261-1270.

Tax Justice Network- Africa (2011), Tax us if you can. Why Africa Should Stand up for Tax Justice. Report.

van Dijk M., Weyzig F., Murphy R., (2006), The Netherlands: A tax haven?, Report. SOMO, Centre for Research on Multinational Corporations, Amsterdam.

Young M. A. (2013), Banking secrecy and Offshore Financial Centers. Money laundering and offshore banking, Routledge, London and NY. 